\title{
Simulation of Automated Visual Inspection Systems for Specular Surfaces Quality Control*
}

\author{
Juan Manuel García-Chamizo, Andrés Fuster-Guilló, and Jorge Azorín-López \\ U.S.I. Industrial Information Technology and Computer Networks \\ Information Technology and Computing Dept. University of Alicante. P.O. Box 99. E-03080. \\ Alicante. Spain \\ \{juanma, fuster, jazorin\} @dtic.ua.es \\ http: //www.ua.es/i2rc
}

\begin{abstract}
This paper proposes the use of simulations as a design mechanism for visual inspection systems of specular surfaces. The system requirements and the characteristics of the objects involve a technological design problem for each of the solutions to be developed. A generic model is proposed. It may be adapted or particularised to solve specific inspection problems using simulations. The method results in a flexible low cost design, reducing the distance between the design model and system implementation in a manufacturing procedure. The proposed simulator generates model-based architectures. The paper shows the results on application of metallized automobile logos.
\end{abstract}

Keywords: automated visual inspection, specular surfaces, simulation, quality control.

\section{Introduction}

This paper presents the use of simulations in order to generate automatically vision systems, in particular, for the visual inspection of specular surfaces. This type of material shows optical difficulties due to have a high reflection coefficient. It causes undesirable reflections and shines that conceals chromatic and morphology information about the object.

Most conventional methods of artificial vision proposed ignore the specular reflection characteristics of materials. They focus on surfaces whose reflection is essentially diffuse, arguing that it contains the object information. However, by making use of traditional techniques, methods have been developed with certain restrictions trying to identify the specular reflection component in order to separate it from the diffuse component [1][2]. Alternatively, specific methods have been developed that make the most of specular reflection to extract the shapes of objects [3][4][5][6][7].

Inspection of products with specular reflection is an open problem. Although some partial solutions have been provided, very few systems have been developed for addressing the automatic visual inspection of these objects. The solutions proposed make use of characteristics of active vision. Lighting of the scene is a determining

* This work was supported by the Spanish MCYT DPI2005-09215-C02-01. 
factor as it is possible to facilitate the detection of defects in images [8]. Structured lighting techniques are considered reliable and adequate for inspection of the surface shape and are presented in the few systems of the literature $[9][10][11][12][13][14][15]$. In addition, the use of the same sensor to determine reflectance is another argument for this type of technique (regularly it should be acquired in an independent manner). The systems differ according characteristics of the pattern: the acquisition of lighting pattern is acquired, through projection on the surface or the attention of the system on the reflection (considering the object as part of the optical system where lighting patterns are projected); the instruments used in generating the pattern [16] (screens, projectors, etc.); and, the method used for codification [17][18].

Efficiency and speed are the two strictest conditions that the inspection technique has to satisfy [15] [19]. Efficiency is related to the vision system accuracy rates. According to speed, the system should operate at the rate of the rest of the production line. These requirements involve a technological design problem for each of the solutions to be developed. In addition, the prototype on which tests and corrections are carried out is physical, which causes an increase in both financial and temporal costs. The selection of working and lighting conditions is complex and critical for a satisfactory inspection [20]. In some works, simulations were carried out in order to select the characteristics of lighting pattern and methods of capture. Nevertheless, the choice of different patterns has not been considered, nor are other factors taken into account which would affect the effectiveness of the system such as the angle of capture, zoom, focus, etc. [11][21][22].

Within this framework, it is proposed to model and methods for vision systems designed to automatic inspection of products with specular reflection surfaces and undefined curvature. The solution consists of minimising the lack of sensitivity that takes place in certain image acquisition conditions (those related with specular surfaces). The inspection model amplifies the relevant characteristics defining the objects by means of environmental conditioning and camera calibration [23].

The inspection architectures of a specific product may be conceived through particularisation of the general model. To achieve this, it is proposed to develop a simulator. It will enable to validate the model and guide the experiments using laboratory instruments. The simulator will enable evaluation of the architectures as an alternative to preliminary tests on the physical prototypes.

The basic contribution of this work focuses on the general nature of the inspection model. It will enable architectures to be developed through particularisation using simulations. This method will permit a reduction of distance between the stages of hypothesis and experimentation. That is, a reduction of the technical or scientific development cycle by means of preliminary validation of the viability of the model, and reducing experimental costs by using virtual prototypes.

\section{Model for Inspection of Specular Surfaces}

The inspection model is based on a normalisation $\Upsilon$ that determines the different defects in an image. The transformation $\Upsilon$ uses two stages (see figure 1): $\Upsilon^{\mu}$ and $\Upsilon_{e, c}$. The transformation $\Upsilon^{\mu}$ is able to determine the function $\mu$ of the object (that is 
chromatic, morphological, topographical information, etc.) and will be the objective of the inspection system. Establishing $\Upsilon^{\mu}$ is a complex transformation when specular surfaces take part in the scene. Therefore, performing transformation $\Upsilon_{e, c}$ will enable us to obtain an image in which the sensitivity is adequate to perceive the defects. The problem is solved by obtaining the precise expression for $\Upsilon$ in each case. However, the aim of providing a general solution motivates the decision to resort to explicit forms in order to embody the knowledge contained in $\Upsilon$.

The transformation $\Upsilon_{e, c}$ stores the suitable environmental and calibration conditions in order to obtain an improved image and to be able to determine the defects: lighting angles, vision angles, focus distance, chromaticity, polarisation and other characteristics of light transmission.

It is known that the camera provides a radiance measurement for the scene [24]. Assuming that the inspection object is unique in the scene, the scene radiance, $L_{R}$, is related to the object reflectance (Bidirectional Reflectance Distribution Function, $\mathrm{BRDF}), f_{R}$ [24], and irradiance, $I$, which affects it according to the equation (1).

$$
L_{R}=f_{R} I
$$

The environment modulates the contribution of the object in the camera. To be precise, as can be seen in equation (1), the irradiance, function of the environment, $I$ modulates the object reflectance $f_{R}$ in $L_{R}$ (captured by the camera). Thus, without considering the other perception characteristics, irradiance is a key factor since it enables areas of the surface of the object to be isolated, the contrast in the camera to be increased or decreased, etc.

Therefore, with the aim of reducing the cost of determining the conditions in which the capture of objects has to be performed, an irradiance function $I$ for the object is originally established: $\Upsilon_{e \Phi}$. This function acts as an amplifier of the reflectance of the object, and consequently of the sensitivity of the sensor in order to increase the ranges of environmental and calibration conditions where the system is able to perceive the defects. In this way, the system can perceive the defects in more values of angles conditions, resolutions, etc.

Transformation $\Upsilon_{e \Phi}$ must allow different areas to be established on the object so that its projection on the image will enable the variations in the object functions to be established in those areas. For this purpose, we propose a lighting scheme according to which the adjacent areas of the object will be radiated by different spectral powers, defining, in this way, areas of different radiances. These radiances will establish independent areas on the image, which will allow them to be studied individually. Thus, areas with different responses dependent on the radiant energy and on the reflectance $f_{R}$ of the object will be established in the image domain. It is interesting to define the function determining the energy that later reaches the object in terms of the field radiance $\left(L_{f}\right)$ and which can be adapted to the different situations. In order to do this, the function $\Upsilon_{e \Phi}$ is defined according (2).

$$
\Upsilon_{e \phi}\left(m, \mu_{i}\right)=L_{f}(s, \Lambda, \xi, t)
$$

The function consists of four parameters: $s, \Lambda, \xi \mathrm{y} t$. The area of interest in the motive may be configured based on the parameter $s$. This function establishes the 
morphology of the regions, which will form on the surface. The variable $\Lambda$ contains the set of lighting characteristics that cover each of the established regions. The function $\xi$ determines the spatial configuration of the energy that reaches the motive by means of distribution of the lighting characteristics of $\Lambda$ in each of the areas established by the function $s$, which shall be projected on the image. Finally, it is function of the time $t$ : if the structuring of the lighting is temporal, it will be necessary to generate a sequence of patterns.

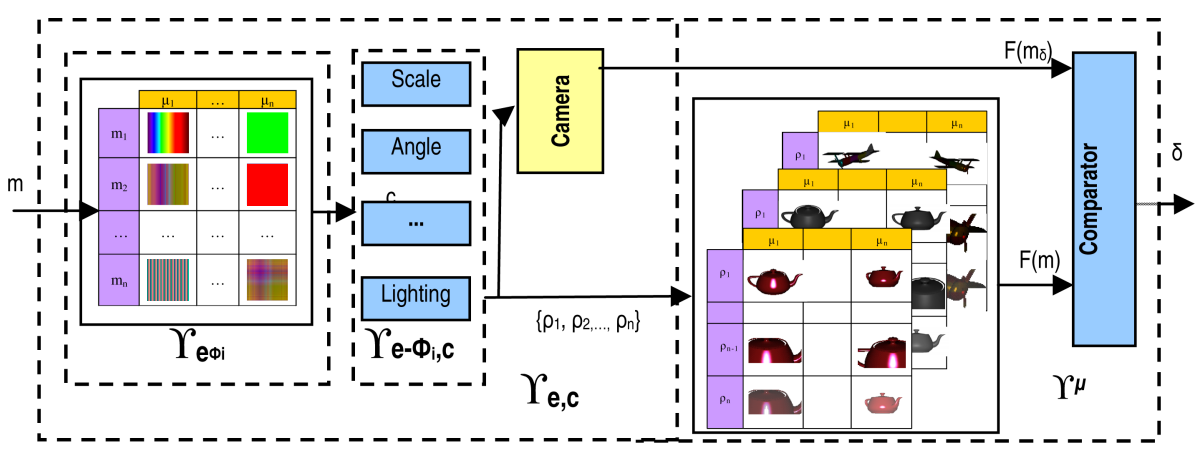

Fig. 1. Inspection model based on the input of a motive $m$, transformation $\Upsilon_{e, c}$ will determine the environmental and calibration conditions so that the camera can capture it. Prior to this, the motive reflectance is increased by means of $\Upsilon_{e \Phi}$. Transformation $\Upsilon^{\mu}$ will determine variation $\delta$ of the motive based on the comparison between image $\mathrm{F}\left(m_{\delta}\right)$ and the stored image.

Once the function $\Upsilon_{e \Phi}$ has been defined, it is necessary to establish the rest of conditions of the magnitudes of the scene $\rho_{i}$ (3) where the system is capable of perceiving adequately (we have called this process tuning the system).

$$
\rho_{i}=\rho_{i}(m, e, c)
$$

The magnitudes $\rho_{i}$ contribute to the formation of the image: scale, angle of the camera, lighting intensity, frequency, saturation of color, etc. Each magnitude is a function of the magnitudes $\mu_{i}$ of the motive $m, \varepsilon_{i}$ magnitudes of the environment $e$ and $\gamma_{i}$ of the camera. The contribution of the motive $m$ to the image constitutes the really valuable information. Therefore, camera and environment variables will need to be established $\Upsilon_{e-\Phi, c}$ for different defects which may be produced in the object.

Finally, the comparison stage $\Upsilon^{\mu}$ is established between the image obtained in the transformation $\Upsilon_{\mathrm{e}, \mathrm{c}}$ and the image stored in the knowledge base. The inspection stage determines the image processing techniques, which will enable detection of defects in the inspection motive. For example, a series of descriptors may be established for the inspection images and those stored in the database in order to compare them. It is also possible to differentiate on the same image but requires a transformation or rotation against the motive, environment, etc. A graphic diagram of the transformation process may be observed in figure 1 . 
The specification of each transformation $\Upsilon$ establishes the computational and economic costs of the inspection system for the detection of defects in surfaces of specific products. The design of the architectures will be required to consider performance and cost criteria, measured in relation to robustness, accuracy, density of information number of images and speed among other items.

\section{Simulation Aided Design}

Simulation provides a mechanism for preliminary validation of technological designs and assistance in conception models. It may be used to reinforce, reject or redirect the basic hypothesis of research. It has a direct consequence on costs associated to each of the stages of scientific method and, mainly, in the laboratory experimentation. Computer simulation enables controlled tests to be carried out on initial hypothesis facilitating refinement until it is mature. A systematic flexible low cost validation method is established without any need to perform preliminary experiment on physical prototypes.

Computational modelling of systems and their simulation as validation mechanisms is not a new idea. Computer simulation has been introduced in almost all areas of scientific and technological knowledge. For example, in the technological field, simulations are carried out in order to contrast ideas of a potential system prior to addressing its design. However, this is not a systematic procedure.

Computer simulation may become a universal and flexible tool that can be used systematically in scientific analyses or technological designs. It provides a rapid abstraction and specification mechanism based on computational models. It would permit progress in all economic, social, industrial sectors, etc. For example, the advantages associated to assisted design through simulation of the inspection system primarily consist of: a substantial cost reduction in terms of system analysis time and its development in the production line; reduction of financial costs of the technological material required, as the process obviates the need for expensive physical prototypes; maintenance of knowledge bases of inspection systems with respect to their technology, processing modules and, in general, the designed architecture which could be used for implementing other similar systems in respect of inspection product specifications; and finally, reduction of errors deriving from the system analysis phase, design and implementation of real inspection systems, which may be quickly detected before considerable costs are incurred.

In the observation stage of scientific or technological development, the introduction of computational models will enable virtual worlds to be established based on scientific or technological models and theories. Some of the advantages of the use of virtual worlds are the reduction of costs associated to observation, laboratory material costs and the possibility of generating data repositories in a rapid and flexible manner.

In the experimentation phase, the computational models developed would be able to guide the experiments to be carried out. Laboratory experiments usually use a restricted universe model. In many cases, this is artificial, designed ad hoc in order to carry out the appropriate tests to validate basic hypothesis. This could be substituted by a computational model. In some cases, it may even be more appropriate for the 
experimentation phase. For example, Hughes argues for the inclusion of algorithms that simulate complex physical systems in order to carry out experiments [25]. Norton and Suppe defend simulation as a means of experimentation, which serves as an instrument to test or detect phenomena in the real world [26]. The difficulty of experimentation on a conventional model may exceed the material possibilities available at any given time. For example, studies on human knowledge and intelligence have been more promising, by applying computational models which use the brains of certain animals as a human model, as we all know.

\section{Simulation of Model-Based Architectures}

The computational modelling required to simulate hypotheses should affect several levels: from image formation, based on scene magnitudes, to the visual process of automatic inspection based on the $\Upsilon$ transformations proposed. In the first stage, a virtual environment is developed based on existing models and scientific theories in order to form the image. Later, computational modelling is established for the hypothesis of the visual inspection system for specular surfaces.

\subsection{Modelling of the Inspection System Hypothesis}

The simulator stage related to $\Upsilon$ transformation recreates the necessary characteristics for inspection the virtual world generated based on the synthesis of magnitudes of the scene $\rho_{i}$ (scale, angle and lighting) and synthesis of the image. In addition, it takes into account performance $P$ and cost $C$ offered by the architecture of the vision system. They are additional characteristics that will facilitate study experimental data and extract conclusions in the automatically generation of hypotheses for vision system architectures. The performance $P$ is related to the efficiency $E$ and speed (the two restrictive conditions which must be fulfilled by the inspection system) of the system for inspection of a magnitude $\mu_{\mathrm{i}}$ of motive $m$. The speed will be studied through the inspection time $T$. Cost $C$ is related to the technological market aspects implicated in obtaining the architecture.

The computational model ${ }^{*} \Upsilon_{e \Phi}$ (4) of transformation $\Upsilon_{e \Phi}(2)$ stores, in knowledge bases for each of the magnitudes $\mu_{\mathrm{i}}$ (chromaticity, morphology, topography, etc.) to be inspected: the function of the field radiance $L_{f}$, performance $P_{\mathrm{e} \Phi}$ and the cost $\mathrm{C}_{\mathrm{e} \Phi}$ associated to the architecture of the inspection system using this radiance. The contribution of performance $P_{\mathrm{e} \Phi}$ to the global $P$ of the architecture reflects the time $T_{\mathrm{e} \Phi}$ taken by the system establishing radiance $L_{f}$ and efficiency $E_{\mathrm{e} \Phi}$ that provides this structured lighting. The cost $\mathrm{C}_{\mathrm{e} \Phi}$ is associated, in this case, to the costs of elements that enable the desired structured lighting to be obtained: sources of lighting, elements of positioning, etc.

$$
{ }^{*} \Upsilon_{e \Phi}\left(m, \mu_{i}\right)=\left\langle L_{f}(s, \Lambda, \xi, t), P_{e \Phi}, C_{e \Phi}\right\rangle
$$

Transformation $\Upsilon_{e-\Phi . c}$ establishes the appropriate tuning of the system (scale, angle lighting, etc.) in order to determine any defects that might appear in the various magnitudes $\mu_{\mathrm{i}}$ of the motive $m$. It will determine the appropriate values of $\rho_{i}(3)$ for 
the system in terms of its components (environment and camera). The system tuning necessitates inferring in any of the variables intervening in the magnitude of the associated $\rho_{i}$ scene (we have considered for the inspection problem the scale $\rho_{E}$, angle between the camera and the motive $\rho_{\theta}$ and lighting intensity $\rho_{I}$ ). In the case of the scale $\rho_{E}$ the system will position the motive at a specific distance from the camera or at the same distance will position the camera from the motive or change the zoom camera. The procedure is similar in the case of the angle $\rho_{\theta}$, the motive or the camera may be positioned and oriented. In the case of tuning the lighting $\rho_{I}$, all environmental parameters $e$ and the camera $c$ will be used. The variables which permit conformation of values of the desired magnitudes of the scene $\rho_{i}$ establish the tuples $G \rho_{E}, G \rho_{\theta}, G \rho_{I}$ for the scale, the angle and the lighting respectively.

The computational model for ${ }^{*} \Upsilon_{e-\Phi . c}$ (5) makes use of knowledge bases which store a set of tuples $G \rho_{E}, G \rho_{\theta}$ and $G \rho_{I}$ for the $\rho_{i}$ values suitable for inspection of the complete motive. Also, performance $P_{e-\Phi . c}$ and associated $\operatorname{cost} C_{e-\Phi . c}$ in a similar way to the function ${ }^{*} \Upsilon_{e \Phi}$ is considered.

$$
{ }^{*} \Upsilon_{e-\Phi, c}\left(m, \mu_{i}\right)=\left\langle\left\{G \rho_{E}, G \rho_{\theta}, G \rho_{I}\right\}, P_{e-\Phi, c}, C_{e-\Phi, c}\right\rangle
$$

The temporal cost $T_{e-\Phi, c}$ of performance $P_{e-\Phi . c}$ depends on the variables chosen to obtain the value $\rho_{i}$. For example, the cost $T_{e-\Phi, c}$ of establishing a scale value $\rho_{E}$ based on a change in the zoom of the optic may be less than doing so by means of a repositioning of the motive or of the camera. The cost $C_{e-\Phi . c}$ will be given by the technological material that enables to obtain the magnitudes of the scene associated to the scale $\rho_{E}$, the angle $\rho_{\theta}$ and the lighting $\rho_{I}$.

The transformation " ${ }^{\mu}$ will determine the defects of the inspection motive establishing the efficiency of the system $E_{\mu}$. Time $T_{\mu}$ required for motive inspection and the $\operatorname{cost} C_{\mu}$ of the implementation determine the final stages of the architecture.

$$
\begin{aligned}
& E(m, \mu)=E_{e \Phi}=E_{e-\Phi, c}=E_{\mu} \\
& T(m, \mu)=\left\{T_{e \Phi}, T_{e-\Phi, c}, T_{\mu}\right\}
\end{aligned}
$$

The performance $P$ of the architecture for the inspection of a magnitude $\mu$ of the motive $m$ will therefore be defined according to equation (6). The efficiency $E$ of the architecture will be stored in each transformation ${ }^{*} \Upsilon_{e \Phi},{ }^{*} \Upsilon_{e-\Phi . c}$ and ${ }^{*} \Upsilon^{\mu}$. The time $T$ for the inspection will depend on the time for establishing the radiance $\mathrm{T}_{\mathrm{e} \Phi}$, for the tuning the magnitudes of the scene $\rho_{i}, T_{e-\Phi, c}$, and for the inspection $T_{\mu}$.

Finally, the cost of the architecture $C$ (7) will depend on the technological cost of all the implicated elements.

$$
C(m, \mu)=C_{e \Phi}+C_{e-\Phi, c}+C_{\mu}
$$

\subsection{Generation of Architecture Hypotheses for Inspection}

Let $A$ set of possible architectures for visual inspection of a set of motives $M_{I}(8)$. An architecture $a$ is defined as an ordered set of tuples which contain the characteristics 
for carrying out transformations $\Upsilon_{e \Phi}$ and $\Upsilon_{e-\Phi, c}$. This ordered set contains the work plan for inspection of $M_{I}$.

$$
A=\left\{a=\left\{\left\langle\Upsilon_{e \Phi}\left(m_{i}, \mu_{j}\right), \Upsilon_{e-\Phi, c}\left(m_{i}, \mu_{j}\right)\right\rangle\right\}: \cup\left(m_{i}\right)=M_{I}\right\}
$$

Therefore, the order of the motives $m$ to be inspected, the radiance conditions (4), and tuning of the magnitudes of the scene $\rho_{i}$ define the characteristics of the system. They establish performance $P$ and cost $C$ for the inspection of a set of motives. The choice of architecture $a$, of set $A$, of those possible for inspection of $\mathrm{M}_{\mathrm{I}}$ will be determined by different criteria relating to that performance $P$ and cost $C$ : reduction of costs, increased performance, increased ratio performance/cost, etc.

The hypothesis generator and simulator of the inspection system should be able to provide the appropriate architecture for each problem. In order to carry out this procedure, the system calculates the performance $P$ and cost $C$ of the architecture in all conditions: for each defect of the motive $m$ and, for all the motives to be inspected, for each of the radiance conditions (according to transformation $\Upsilon_{e \Phi}$ ) and for all the scene magnitudes (scale $\rho_{E}$, angle $\rho_{\theta}$, and lighting $\rho_{I}$ ) defined for the problem study.

In this work, we focus specifically performance $P$ of the system, in particular, the efficiency $E$. Time $T$ and cost $C$ depend on aspects of the technology at any given time. We propose to calculate the difference between the images of a defective motive $m_{j}$ and a motive without defects $m_{i}$ (model motive) in order to extract the defects $m_{\delta}$ (9) in the same conditions. The difference is in accordance to specified transformation $\mathrm{Y}^{\mu}$ and is designed to be an independent measurement of any other transformation that may be chosen ad hoc.

$$
F\left(\rho\left(m_{\delta}, e, c\right)\right)=\left|F\left(\rho\left(m_{i}, e, c\right)\right)-F\left(\rho\left(m_{j}, e, c\right)\right)\right|
$$

In addition, in order to ensure that the difference between the images is independent of capture, the defective motive $m_{j}(10)$ is provided with a series of defects $m_{\delta}$, in each $\mu_{i}$, distributed uniformly over the surface of the motive. A function of defects synthesis syndef is applied to the model motive $m_{i}$.

$$
m_{j}=\operatorname{syndef}\left(m_{i}, m_{\delta}\right)
$$

The ratio between the number of points in the difference image (9) and the number of points which would represent defects $T(m, e, c)$ provide the efficiency $E$ of transformation $\mathrm{\Upsilon}^{\mu}$. We use this calculation as a measurement of sensitivity of the system, of the capacity for perceiving defects, and it will be stored in a knowledge base $\mathrm{BD}_{\delta}(11)$. We have called $\mathrm{BD}_{\delta}$ sensitivities database.

$$
B D_{\delta}\left(m, \mu_{i}, \Upsilon_{e \Phi}, \rho_{E}, \rho_{\theta}, \rho_{I}\right)=\frac{\#\left(F\left(\rho\left(m_{\delta}, e, c\right)\right)\right)}{\# T\left(m_{\delta}, e, c\right)}
$$

Sensitivities database $\mathrm{BD}_{\delta}$ queries will enable us to construct transformations ${ }^{*} \Upsilon_{e \Phi}$ and ${ }^{*} \Upsilon_{e-\Phi . c}$ according to their performance $P$. Consequently, the magnitudes $\rho_{i}$ shall be determined in which the system sensitivity is appropriate for the perception of defects. 


\section{Inspection of Automobile Logos}

This section presents the results of the application of the model and the simulator to the development of specular automobile logo inspection systems based on the efficiency $E$ of the system. Firstly, the virtual environment was developed based on existing scientific models and theories for image formation. In order to carry out the study of local light reflection, the reflectance model presented by Cook-Torrance [27] was used as a basic element. Recent studies show its considerable ability to adapt to reflectance extracted from objects [28].

\subsection{Creation of the Sensitivities Database}

For the creation of the sensitivities $\mathrm{DB}_{\delta}(11)$, planes were used in order to control the angle formed by the elements involved in the scene on all parts of the object. Three types of defects were distributed over these $12 \times 12 \mathrm{~mm}^{2}$ planes: two changes in topography (a $0.6 \mathrm{~mm}$-diameter crack or crater) and a change in colour on a surface measuring $0.6 \times 0.6 \mathrm{~mm}^{2}$. The planes have a specularity coefficient (s in CookTorrance model [27]) of 0.75 and a roughness (rms, or $\mathrm{m}$ in [27]) of 0.1 . There are two types of planes with the above characteristics: dielectric and metallic. The dielectric planes have a refraction index of 1.6, while the metallic planes have a refraction index of 2.8 and an extinction index of 3.2 using Fresnel equations.

a)

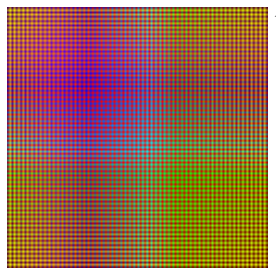

b)

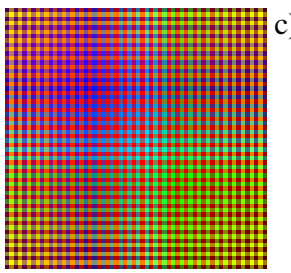

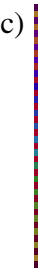
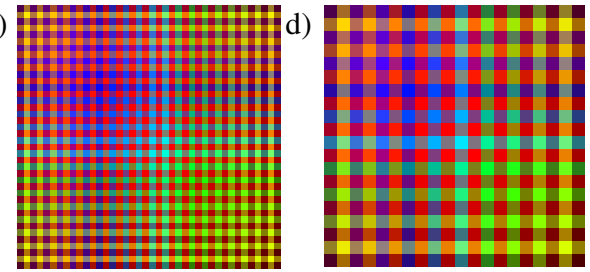

Fig. 2. Lighting environmental conditioning Interlaced $X Y$ with different areas for interest. a) $0.1 \times 0.1 \mathrm{~mm}^{2}$ b) $0.2 \times 0.2 \mathrm{~mm}^{2}$ c) $0.3 \times 0.3 \mathrm{~mm}^{2}$ d) $0.6 \times 0.6 \mathrm{~mm}^{2}$

A function of environmental conditioning $\Upsilon_{e \Phi}$ that provides maximum sensitivity of the system to perceive the defects Interlaced $X Y$ [23] and a non-structured lighting function Homogeneous (white lighting) were taken into account. The goal is to show the influence of the increase in perception capacity using $\Upsilon_{e \Phi}$ and the development of inspection architectures according different sensitivity results for each type of material (dielectric or metallic). The function $\xi$ of the Interlaced $X Y$ lighting distribute monochromatic lights $\Lambda$ of different set of wavelengths $(380-780 \mathrm{~nm})$ in different regions. The adjacent regions of a certain region are different maximizing the differences of the wavelengths between them (see figure 2). There are different regions of interest for each of these functions, $\left(0.1 \times 0.1 \mathrm{~mm}^{2}, 0.2 \times 0.2 \mathrm{~mm}^{2}, 0.3 \times 0.3\right.$ $\mathrm{mm}^{2}$ and $0.6 \times 0.6 \mathrm{~mm}^{2}$ ). Time $t$ is not considered in this case. 
The system tunes the magnitudes of the scene $\rho_{i}$ modifying camera angles with respect to the normal object $\left(0^{\circ}-90^{\circ}\right)$, the scale $\rho_{E}(1 \mathrm{p} / \mathrm{mm}, 5 \mathrm{p} / \mathrm{mm}, 10 \mathrm{p} / \mathrm{mm}$ and 15 $\mathrm{p} / \mathrm{mm})$ and the lighting angle $\left(0^{\circ}-90^{\circ}\right)$ with respect to the normal to the object in order to consider different lighting values $\rho_{\theta}$.

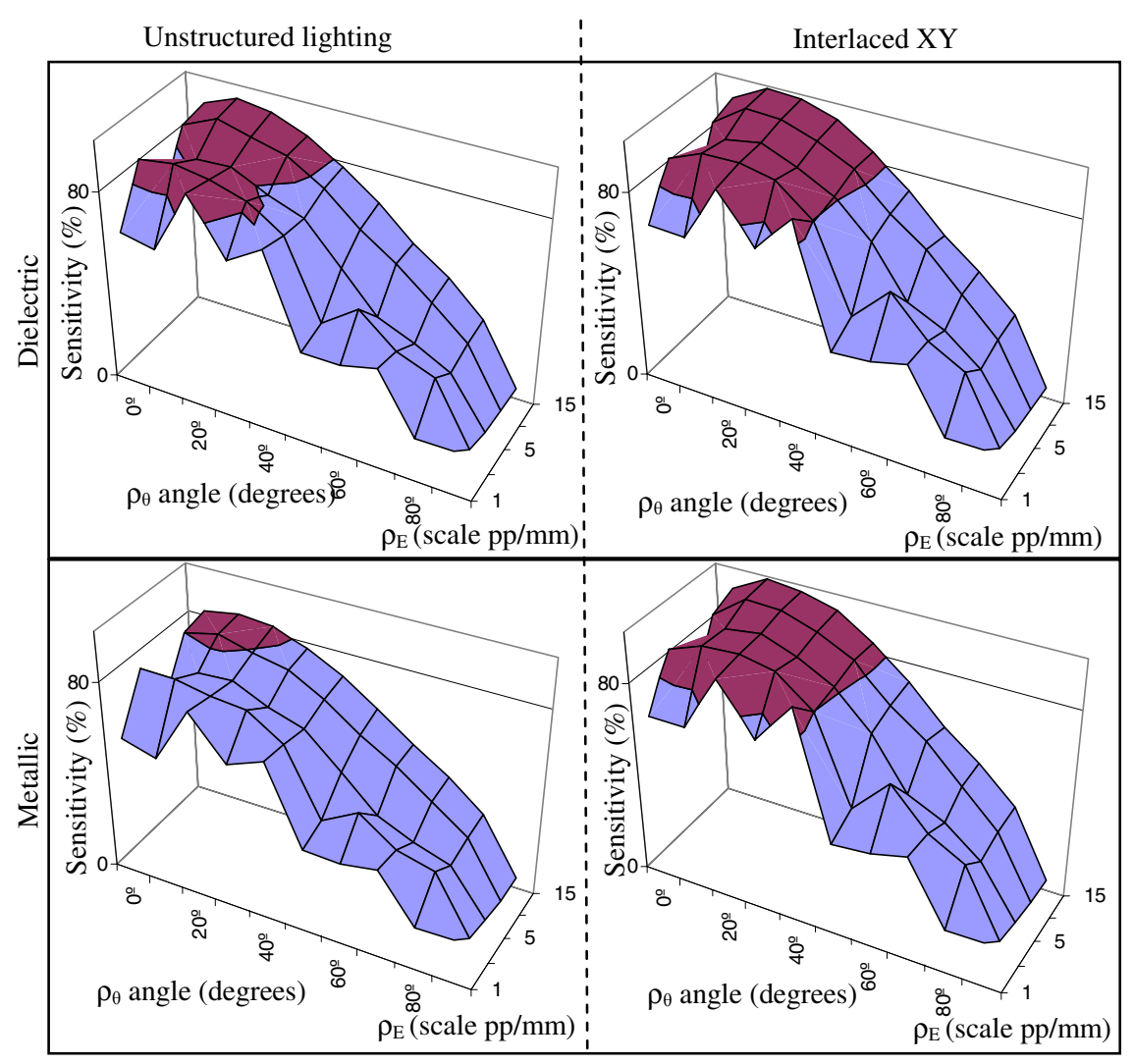

Fig. 3. Partial view of the $\mathrm{BD}_{\delta}$ for dielectric and metallic motives according to lighting configuration $\Upsilon_{e \Phi}$ unstructured and used for amplification of the sensitivity (Interlaced XY)

Figure 3 shows partial views of the database $\mathrm{DB}_{\delta}$ organised according to the scale $\rho_{E}$, angle $\rho_{\theta}$ and motive characteristics. The average value was used for the remaining characteristics such as the areas of lighting, types of defects, lighting scene $\rho_{I}$, etc.

\subsection{Application of the Mercedes-Benz $z^{1}$ Logo Inspection Results}

We have used a Mercedes Benz logo with a $100 \mathrm{~mm}$ diameter in order to show the application of results on the inspection of specular surfaces. The geometric characteristics of the part define a total inspection surface of $4676.15 \mathrm{~mm}^{2}$.

\footnotetext{
${ }^{1}$ Mercedes Benz and its logo are a trademark of DaymlerChrysler AG.
} 
Given that the minimum sensitivity threshold for detection depends on the application, in this case a minimum rate of sensitivity in the inspection of $80 \%$ has been assumed.

The inspection of a dielectric motive with lighting of the Homogeneous type can be carried out in 15 points of the scene magnitudes $\rho_{i}$ (see figure 3 ). The environmental conditioning of the measure providing the function of maximum sensitivity, Interlaced $X Y$, raises the number of $\rho_{i}$ points to 19 , where it is possible to perceive adequately the defects.

The inspection of a metallic motive with an unstructured lighting environment restricts the inspection conditions to only five values of the scene magnitudes $\rho_{i}$ (this is a situation of less sensitivity than those studied in the conditioning experiments [23]). The results contrast with those achieved by $\Upsilon_{e \Phi}$ Interlaced $X Y$, which permits an adjustment of the scene magnitudes system by 20 points (figure 3 ).

The choice of conditions in which capture for inspection of the whole part is carried out is a complex problem. It is necessary to take into account the particularities of each solution. In this case, in order to apply the results, the scale $\rho_{E}$ has been set at its greatest value (15 pixels per millimetre). Variations may be given in the perception angles $\rho_{\theta}$ (angle between the normal to the camera and the normal to the motive) using the lighting function of the Homogeneous type will be up to $30^{\circ}$, for dielectric surfaces, and up to $20^{\circ}$, for metallic surfaces. In the case of using the best conditioning function, Interlaced $X Y$, the angles may vary between $0^{\circ}$ and $40^{\circ}$, irrespective of the electromagnetic characteristics. In order to determine the appropriate angles between camera and motive, an approximation to the optimum solution was proposed which increases the area of inspection of the parts and reduces the images to be captured. For this purpose, a search tree was designed using a branch and bound algorithm, in which the solutions space of each node is reduced to a maximum of five children and a maximum temporal processing level is established.

The sensitivity associated to the values of the scene magnitudes conditions the capture of the motive in 15 images for inspection of this part with a surface of electromagnetic characteristics of the dielectric material and using a Homogeneous lighting configuration.

The solution extracted from the search tree that maximises the area to be inspected and reduces the number of captures, is able to process a total surface of $4671.17 \mathrm{~mm}^{2}$, practically $100 \%$ of the surface. The resolution requirements of the camera vary from $740 \times 740$ to $1452 \times 1452$ pixels of the first capture (figure 4 ).

Inspection of the dielectric part using maximum sensitivity environmental conditioning, function $\Upsilon_{e \Phi}$ Interlaced $X Y$, reduces the capture set of 15 to 9 images. The camera resolution varies between $826 \times 826$ and $1455 \times 1455$ pixels in order to cover a total surface area of $4675.05 \mathrm{~mm}^{2}$. The first capture obtains $45 \%$ of the total inspection of the logo. This fact contrasts with that carried out with the unstructured lighting environment that, with the same position, permits only $11.58 \%$ of the part.

The metallic logo inspection requires 26 captures using Homogeneous type lighting. In this case, angles are only permitted between the camera and the normal angles to the motive of less than $20^{\circ}$, setting a scale of perception of 15 pixels per $\mathrm{mm}$. Thus, the inspection plan is the worst. With a camera of $1452 \times 1452$ pixels, it 

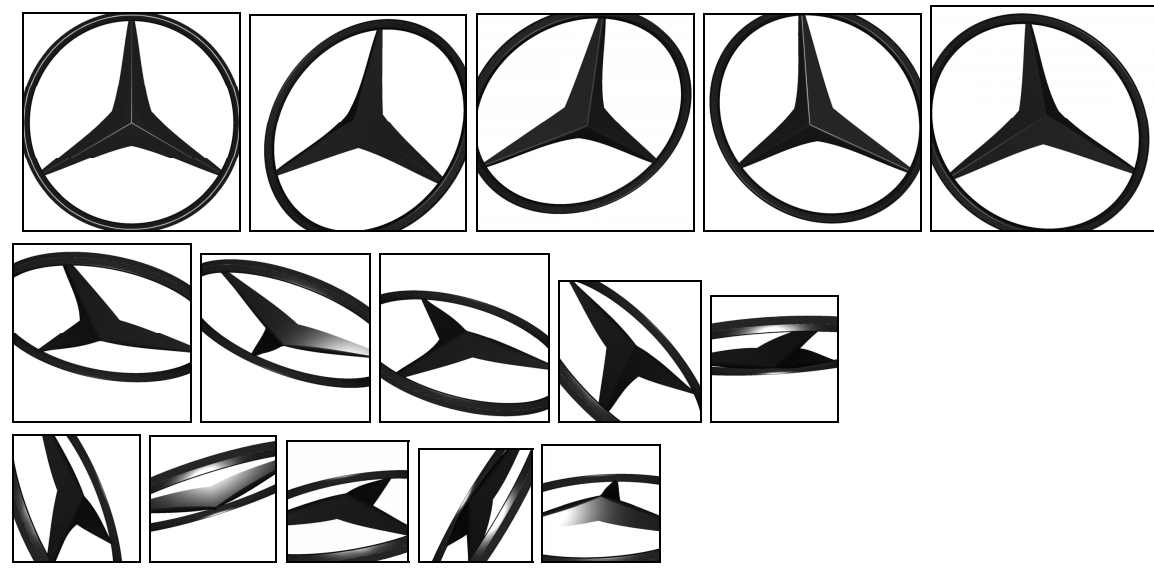

Fig. 4. Captures of the Mercedes Benz dielectric logo using the Homogeneous function Ye $\Phi$
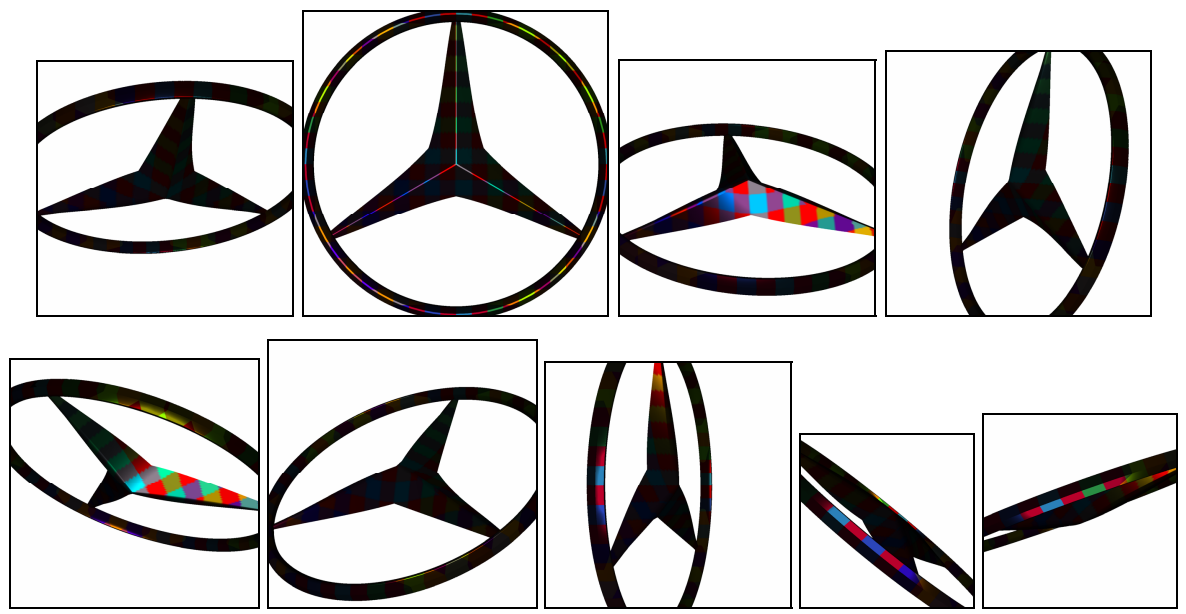

Fig. 5. Captures using the function $\Upsilon e \Phi$ Interlaced $X Y$ for the metallic logo

is able to inspect a total surface area of $4619.96 \mathrm{~mm}^{2}$, which represents $98.79 \%$ of the part. Certain captures may be made a lower resolution of up to 510x510 pixels.

Finally, the inspection of the metallic surface using the structured lighting that provides function $\Upsilon_{e \Phi}$ Interlaced $X Y$ produces a considerable improvement in the number of captures from 26 to 9 (see figure 5).

\section{Conclusions}

The research addresses the problem of the adverse conditions vision in which specular surfaces intervene. A general model is provided in which solutions to problems may 
be obtained by particularisation on a formal framework that enables study in the domain of the motive. The use of simulators for validation of hypotheses reduces the distance between abstract notions and reality of the phenomena. It provides research with a reduction of financial costs in the requisite technological material, reducing the need for expensive economic prototypes, whilst also rationalising development times.

Automatic generation and simulation of the system hypotheses for inspection using computational models has a direct effect on the reliability and cost of such systems. The study enables the best conditions for carrying out the inspection of specular surfaces to be discerned through evaluation of the proposed architectures: with respect to resolution and capture angles, lighting of the environment, etc. These may be reasoned and deliberated before being put into practice. The conclusions of the simulation will establish for example, the minimum characteristics of the system, avoiding particularly complicated technological designs that do not necessarily lead to increased performance.

Simulation confirms the basic hypothesis of the work, and it would be appropriate to further advance the physical experiment by framing the technological development of visual inspection for specular surfaces for specific industries: the automobile industry, bathroom fixtures and fittings, jewellery, marble, mirror manufacturing etc.

\section{References}

1. Swaminathan, R., Kang, S.B., Szeliski, R., Criminisi, A., Nayar, S.K.: On the Motion and Appearance of Specularities in Image Sequences. In: Heyden, A., Sparr, G., Nielsen, M., Johansen, P. (eds.) ECCV 2002. LNCS, vol. 2350, Springer, Heidelberg (2002)

2. Lin, S., Shum, H.: Separation of Diffuse and Specular Reflection in Color Images. In: Proc. of IEEE Conference on Computer Vision and Pattern Recognition, CVPR 2001 (2001)

3. Bhat, D.N., Nayar, S.K: Stereo and specular refection. International Journal of Computer Vision 26(2), 91-106 (1998)

4. Schultz, H.: Retrieving Shape Information from Multiple Images of a Specular Surface. IEEE Transactions on Pattern Analysis and Machine Intelligence 16(2) (1994)

5. Oren, M., Nayar, S.K.: A theory of specular surface geometry. International Journal of Computer Vision 24(2), 105-124 (1997)

6. Svarese, S., Perona, P.: Local Análisis for 3D Reconstruction of Specular Surfaces. In: Proc. of IEEE Computer Society Conference on CVPR 2001, IEEE Computer Society Press, Los Alamitos (2001)

7. Ragheb, H., Hancock, E.R.: A Probabilistic Framework for Specular Shape-form-Shading. In: Proc. 16th International Conference on Pattern Recognition, ICPR 2002 (2002

8. Pernkopfa, F., O'Leary, P.: Image acquisition techniques for automatic visual inspection of metallic surfaces. NDT\&E International, Elsevier 36, 609-617 (2003)

9. Perard, D.: Automated visual inspection of specular surfaces with structured-lighting reflection techniques, Fortschritt-Berichte VDI, vol. 8 (869). VDI Verlag, Dusseldorf (2001)

10. Puente León, F., Kammel, S.: Inspection of specular and painted surfaces with centralized fusion techniques. Measurement (2006)

11. Seulin, R., Merienne, F., Gorria, P.: Simulation of specular surface imaging based on computer graphics: application on a vision inspection system. Journal of Applied Signal Processing - Special issue on Applied Visual Inspection, EURASIP 2002 7, 649-658 (2002) 
12. Seulin, R., Merienne, F., Gorria, P.: Dynamic lighting system for specular surface inspection. In: Conference on Machine Vision Applications in Industrial Inspection VII, SPIE, vol. 4301, pp. 199-206 (2001)

13. Aluze, D., Merienne, A.F., Dumont, C., Gorria, P.: Vision system for defect imaging, detection and characterization on a specular surface of 3D object. Image and Vision Computing, Elsevier Science 20, 569-580 (2002)

14. Morel, O., Stolz, C., Gorria, P.: Polarization imaging for 3D inspection of highly reflective metallic objects. Optics and Spectroscopy 101(1), 15-21 (2006)

15. Newman, T.S., Jain, A.K.: A Survey of automated visual inspection. Computer Vision and Image Understanding 61(2), 231-262 (1995)

16. Zhang, X., North, W.P.T.: Analysis of 3-D surface waviness on standard artifacts by retroreflective metrology. Optical Engineering 39(1), 183-186 (2000)

17. Hung, Y.Y., Shang, H.M.: Nondestructive testing of specularly reflective objects using reflection three-dimensional computer vision technique. Optical Engineering 42(5), 1343 1347 (2003)

18. Rocchini, C., Cignoni, P., Montani, C., Pingi, P., Scopigno, R.: A Low Cost Optical 3D Scanner. In: Computer Graphics Forum. Eurographics 2001 Conference Proc., vol. 20(3), pp. 299-308 (2001)

19. Malamas, E.N., Petrakis, E.G.M., Zervakis, M., Petit, L., Legat, J.D.: A survey on industrial vision systems, applications and tools. Image and Vision Computing 21, 171188 (2003)

20. García-Chamizo, J.M., Fuster-Guilló, A., Azorín-López, J.: Automatic Generation of Image Acquisition Conditions for the Quality Control of Specular Surfaces. In: IEEE/SPIE 8th International Conference on Quality Control by Artificial Vision (QCAV 2007), Le Creusot, France (2007)

21. Kammel, S.: Automated optimization of measurement setups for the inspection of specular surfaces. In: Machine Vision and Three-Dimensional Imaging Systems for Inspection and Metrology II, Proc. SPIE 4567, pp. 199-206 (2002)

22. Seulin, R., Merienne, F., Gorria, P.: Machine vision system for specular surface inspection: use of simulation process as a tool for design and optimization. In: International Conference on Quality Control by Artificial Vision, Le Creusot, France, vol. 1, pp. 147$152(2001)$

23. García-Chamizo, J.M., Fuster-Guilló, A., Azorín-López, J.: Visual Input Amplification for Inspecting Specular Surfaces. In: Proc. IEEE ICIP 2006, IEEE, Atlanta, United States (2006)

24. Nicodemus, F.E., Richmond, J.C., Hsia, J.J., Ginsberg, I.W., Limperis, T.: Geometrical considerations \& nomenclature for reflectance. NBS Monograph 160, National Bureau of Standards, Washington, D.C. U.S. Department of Commerce (October 1977)

25. Hughes, R.: The Ising Model, Computer Simulation, and Universal Physics. Models as Mediators. Cambridge University Press, Cambridge (1999)

26. Norton, S., Suppe, F.: Why Atmospheric Modeling is Good Science. Changing the Atmosphere: Expert Knowledge and Environmental Governance. MIT Press, Cambridge, MA (2001)

27. Cook, R.L., Torrance, K.E.: A Reflectance Model for Computer Graphics. ACM Transactions on Graphics 1(1), 7-24 (1982)

28. Ngan, A., Durand, F., Matusik, W.: Experimental Analysis of BRDF Models. In: Proc. of Eurographics Symposium on Rendering (2005) 